\title{
eJRIEPS
}

Ejournal de la recherche sur l'intervention en éducation physique et sport

$46 \mid 2020$

Varia

\section{Coopérer en éducation physique : Le Jigsaw, une méthode prometteuse?}

To Cooperate in Physical Education: The Jigsaw, a Promising Learning Method?

Océane Drouet, Grégoire Millet et Vanessa Lentillon-Kaestner

\section{(2) OpenEdition}

Journals

Édition électronique

URL : https://journals.openedition.org/ejrieps/4941

DOI : 10.4000/ejrieps.4941

ISSN : 2105-0821

Éditeur

ELLIADD

\section{Référence électronique}

Océane Drouet, Grégoire Millet et Vanessa Lentillon-Kaestner, « Coopérer en éducation physique : Le Jigsaw, une méthode prometteuse? », eJRIEPS [En ligne], 46 | 2020, mis en ligne le 01 avril 2020, consulté le 18 mars 2022. URL : http://journals.openedition.org/ejrieps/4941 ; DOI : https://doi.org/ 10.4000/ejrieps.4941

Ce document a été généré automatiquement le 18 mars 2022.

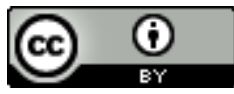

La revue eJRIEPS est mise à disposition selon les termes de la Creative Commons Attribution 4.0 International License. 


\section{Coopérer en éducation physique : Le Jigsaw, une méthode prometteuse?}

To Cooperate in Physical Education: The Jigsaw, a Promising Learning Method?

Océane Drouet, Grégoire Millet et Vanessa Lentillon-Kaestner

\section{Introduction}

1 La pédagogie est à la fois l'art de transmettre et d'émanciper (Meirieu, 2013). Diverses stratégies sont utilisées par les enseignants pour transmettre et émanciper les élèves. Cette diversité d'utilisation s'explique en partie par les conceptions d'enseignement liées directement aux croyances et valeurs des enseignants. Les plus sensibles aux valeurs d'une école humaniste pourront, par exemple, chercher à former leurs élèves à la coopération, à la solidarité, à la réflexion critique et à ce qu'ils comprennent les enjeux fondamentaux du monde contemporain. Les compétences sociales sont particulièrement mises en avant avec la réforme française de l'école de 2015. Les pédagogies coopératives permettent de développer ces compétences en faisant vivre les élèves ensemble, et en les faisant apprendre collectivement. Le respect, l'entraide, le fairplay sont des axes de travail ancrés dans le quotidien des praticiens et pratiquants d'éducation physique et sportive (EPS). Ainsi mettre en place des approches coopératives peut être un levier pour faciliter un climat motivationnel serein, l'engagement, l'apprentissage et l'acquisition des compétences sociales des élèves en EPS.

\subsection{Pédagogies actives et apprentissage coopératif}

2 Dès le début du XXe siècle, des acteurs précurseurs se sont intéressés aux pédagogies coopératives (Baudrit, 2005). Dès lors, des praticiens comme Dewey dès 1927 voient la coopération comme un moteur de l'éducation. D'autres, comme Makarenko, dans les années 30, partagent ses idées sur l'organisation collective où les rôles et les tâches sont en rotation permanente. Hameline (1994) précise que « le partage des tâches et des 
responsabilités permet de réaliser une entraide effective » (p. 188). L'école se calquant sur l'évolution de la société, le pédagogue Freinet voit à travers les coopératives ouvrières la coopération à l'école (Baudrit, 2005), pensée alors comme une communauté de vie où « les élèves peuvent vivre ensemble, travailler ensemble » (p. 13). D'autres acteurs, notamment des chercheurs, réfléchissent également à l'apport de la coopération à l'école. Piaget (1969) par exemple voit la coopération comme « une mise en commun des opérations de chacun » (p. 106). Et c'est un siècle auparavant que s'est développé l'enseignement mutuel consistant en la réciprocité de l'enseignement entre les écoliers, le plus capable servant de maître à celui qui l'est le moins (Hamel, 1818).

3 Les méthodes actives existant depuis le début du $19^{\mathrm{e}}$ siècle mettent « au premier rang la recherche en commun (travail en équipe) et la vie sociale des élèves (selfgovernment)» (Piaget, 1971/1988, p. 115 cité par Baudrit, 2005, p. 13). Des éléments clefs favorisant la coopération apparaissent alors progressivement avec les pratiques et les recherches. C'est plus tardivement que le nom d'apprentissage coopératif apparait dans la littérature éducative américaine notamment sous l'influence de la théorie de l'interdépendance sociale de Deutsch (1949). Celui-ci met en avant la curiosité collective et la motivation des élèves. Ce courant repose sur la construction d'un consensus au travers de la coopération et de l'entraide des membres du groupe afin de faciliter les apprentissages (Kardas, Lewek, \& Matyjaszczyk, 2013).

4 Les méthodes d'apprentissage coopératif sont reconnues à partir de cinq éléments essentiels (Johnson \& Johnson, 1989; Johnson, Johnson, Holubec, \& Roy, 1993) : (1) l'interdépendance positive entre les membres du groupe --un membre ne peut réussir si un autre membre ne réussit pas; (2) les responsabilités individuelles où chaque membre du groupe a la responsabilité de partager ses connaissances pour rendre ses pairs plus compétents ; (3) des interactions « face à face » essentielles pour promouvoir le succès de chacun - aide, assistance, soutien, encouragement ; (4) les compétences sociales qui regroupent le développement du leadership, prises de décision, construction d'un climat de confiance, capacité à communiquer, à négocier pour résoudre les conflits ; (5) enfin, le développement d'une dynamique de groupe reposant sur le partage de buts communs pour apprendre et réussir. Slavin (1996) a développé quatre perspectives majeures de l'utilisation de l'apprentissage coopératif: des perspectives motivationnelles, de cohésion sociale, cognitives, et empiriques. Par rapport à cela, il propose un modèle où la cohésion sociale est nécessaire entre les élèves, de même que la motivation à apprendre pour soi, et à faire apprendre les autres (Slavin, 2011). Ainsi pour lui, l'apprentissage n'est accessible que par le contact avec les pairs et le travail de groupe. Le tutorat ainsi que l'évaluation par les pairs sont à mettre en place pour que l'apprentissage coopératif soit véritablement efficace.

Plusieurs études ont montré l'efficacité des méthodes d'apprentissage coopératif sur la réussite et le développement personnel des élèves dans le domaine scolaire (Baudrit, 2005 ; Johnson \& Johnson, 1989, 2000; Johnson, Maruyama, Johnson, Nelson, \& Skon, 1981; Kyndt et al., 2013). En effet, ce type de méthodes facilite la capacité à appliquer et comprendre le contenu. Les interactions entre pairs y sont encouragées ce qui favorise le développement des compétences interpersonnelles et relationnelles (Epinoux, 2014; Kagan \& Kagan, 2009). Par ces cinq critères essentiels, l'apprentissage coopératif peut engendrer un engagement plus intense dans les tâches d'apprentissage. Cet engagement a des effets sur le développement des élèves notamment en EPS où Casey et Goodyear (2015) ont noté des bénéfices sur l'amélioration de la santé psychologique 
des jeunes par une augmentation de l'estime de soi, du sentiment de compétence ou encore de la motivation. Des bénéfices moteurs ont été mis en avant également (Casey \& Goodyear, 2015 ; Lafont \& Winnykamen, 1999 ; Legrain \& Heuzé, 2006 ; Slavin, 1986). Les dispositifs coopératifs semblent se dessiner comme des pratiques pédagogiques prometteuses (Dyson \& Casey, 2013), le Jigsaw (signifiant puzzle) en fait partie (Aronson, 1978).

\subsection{Les caractéristiques du Jigsaw}

L'origine de la méthode Jigsaw, provient des observations d'Aronson (1978) sur l'agressivité des élèves entre eux alimentée par l'environnement compétitif de la classe. Suite à ces observations, il souhaite désamorcer les situations négatives et réduire les préjugés intergroupes dans les écoles (Williams, 2004). Aronson réfléchit alors à une méthode rendant chaque élève compétent et autonome, et à la fois dépendant de ses camarades pour apprendre et progresser. Cette méthode a pour but de contribuer à une atmosphère sereine et positive où chaque élève a sa place en classe. Dans ce cadre pédagogique, les élèves de diverses origines raciales et ethniques sont forcés de travailler ensemble en partageant un but commun. L'élève apprend pour lui et devient expert d'une compétence dans un premier temps. Puis par la suite, il doit pouvoir transmettre ses compétences et rendre compétents tous les membres de son groupe (Metzler, 2011). À tour de rôle, les élèves sont apprenants et enseignants. Ainsi, l'apprentissage assisté par les pairs est totalement présent dans cette démarche d'enseignement (Peer Assisted Learning, Toping \& Ehly, 1998) ; le tutorat réciproque est utilisé dans ces moments d'échange. Chaque élève est impliqué dans ce procédé dans le but de le rendre actif et engagé.

7 L'utilisation des cinq éléments essentiels de l'apprentissage coopératif fait partie intégrante de la méthode Jigsaw (Metzler, 2011). Sa mise en place en classe s'inscrit dans une structure anticipée et exigeante de quatre étapes. (1) Les élèves appartiennent à un groupe (i.e., groupe d'appartenance). Ces groupes sont de nature hétérogène en leur sein et homogène entre eux et sont constitués de quatre à huit élèves. (2) Les élèves se répartissent dans diverses tâches correspondant à des habiletés spécifiques et appartiennent alors à un groupe d'experts. Chaque élève a alors la responsabilité d'apprendre et d'exécuter cette tâche pour devenir « expert » de celle-ci, et être le plus compétent possible. (3) Chaque élève retourne dans son groupe d'appartenance et a la responsabilité d'enseigner et d'expliquer à ses pairs du groupe d'appartenance la tâche qu'il avait en charge de réaliser, la compétence qu'il avait à travailler dans le but de les rendre compétents à leur tour. (4) Les élèves des groupes d'appartenance travaillent ensemble pour produire un travail final commun; c'est alors un moment d'intégration et d'évaluation. Ainsi tout comme dans un puzzle, chaque pièce - la contribution de chaque élève - est essentielle à l'achèvement du travail (Casey, 2004). Chaque apprenant du groupe de travail coopératif est responsable d'une petite partie de l'apprentissage et l'enseigne aux autres membres, ce rôle le place au centre du processus de création du savoir (Slavin, 2014).

8 Depuis 1978, la méthode Jigsaw a évolué. Désormais, elle comporte plusieurs modèles (Tableau I). Le Jigsaw I est le modèle présenté ci-dessus. Le Jigsaw II est un modèle établi par Slavin (1986) qui vise à renforcer le lien entre l'efficacité du groupe et la performance individuelle en introduisant une structure de récompense de groupe. Les 
élèves reçoivent une note individuelle et une note d'équipe déterminée en ajoutant les résultats des tests de tous les membres de chaque groupe d'appartenance. Les pointages des équipes servent ensuite de base à une compétition entre les groupes d'appartenance. Le Jigsaw III a été développé par Stahl (1994) et évalue le processus d'experts par des questionnaires. Enfin, le Jigsaw IV développé par Holliday (2000) cherche à vérifier l'apprentissage dans les groupes d'experts et d'appartenance sur les compétences par des tests. Les contenus qui ne sont pas encore acquis sont ainsi retravaillés et enseignés par la méthode Jigsaw. Ces tests permettent à l'enseignant de faire un point sur les acquisitions de chaque élève.

Tableau I : évolution de la méthode Jigsaw

\begin{tabular}{|c|c|c|c|c|}
\hline \multicolumn{5}{|c|}{ Variantes de la méthode Jigsaw } \\
\hline Étapes & $\begin{array}{l}\text { Jigsaw I (Aronson, } \\
1978)\end{array}$ & $\begin{array}{l}\text { Jigsaw II (Slavin, } \\
1986)\end{array}$ & $\begin{array}{l}\text { Jigsaw III (Stahl, } \\
\text { 1994) }\end{array}$ & $\begin{array}{l}\text { Jigsaw IV (Holliday, } \\
2000)\end{array}$ \\
\hline 1 & $\begin{array}{l}\text { Groupe } \\
\text { d'appartenance }\end{array}$ & $\begin{array}{l}\text { Groupe } \\
\text { d'appartenance }\end{array}$ & $\begin{array}{l}\text { Groupe } \\
\text { d'appartenance }\end{array}$ & $\begin{array}{l}\text { Groupe } \\
\text { d'appartenance }\end{array}$ \\
\hline 2 & Groupe d'expert & Groupe d'expert & Groupe d'expert & $\begin{array}{l}\text { Groupe d'expert }+ \text { test } \\
\text { de connaissance }\end{array}$ \\
\hline 3 & $\begin{array}{l}\text { Groupe } \\
\text { d'appartenance }\end{array}$ & $\begin{array}{l}\text { Groupe } \\
\text { d'appartenance }\end{array}$ & $\begin{array}{l}\text { Groupe } \\
\text { d'appartenance }\end{array}$ & $\begin{array}{l}\text { Groupe } \\
\text { d'appartenance + test } \\
\text { de connaissance sur les } \\
\text { compétences partagées }\end{array}$ \\
\hline 4 & $\begin{array}{l}\text { Production finale des } \\
\text { groupes } \\
\text { d'appartenance avec } \\
\text { évaluation individuelle }\end{array}$ & \begin{tabular}{|lr} 
Production & finale \\
des & groupes \\
d'appartenance avec \\
test
\end{tabular} & $\begin{array}{l}\text { Production } \text { finale } \\
\text { des groupes } \\
\text { d'appartenance }\end{array}$ & $\begin{array}{l}\text { Production finale des } \\
\text { groupes } \\
\text { d'appartenance }\end{array}$ \\
\hline 5 & & & & $\begin{array}{l}\text { Ré-enseignement de } \\
\text { certaines compétences } \\
\text { si nécessaire }\end{array}$ \\
\hline
\end{tabular}

9 Le Jigsaw demande une rigueur et une préparation enseignante minutieuse. Le rôle de l'enseignant est primordial dans la phase de conception des séquences d'apprentissage, des leçons et des choix des contenus à enseigner. Il est également essentiel dans la phase pédagogique lors de la conduite de ses leçons avec les élèves et les régulations de ces derniers dans les tâches entreprises. L'enseignant a pour objectif de donner aux élèves plus d'autonomie en les impliquant dans la prise de décision pour les faire participer à leur apprentissage ou à celui d'autrui (Jaakkola, Helkkula, \& AarikkaStenroos, 2015). Selon Hänze et Berger (2007), le Jigsaw peut augmenter l'implication, la compétence et l'autonomie des membres du groupe. 


\subsection{La méthode Jigsaw en EPS}

10 L'objectif de l'EPS est de faire vivre une pratique scolaire d'activités physiques, sportives, artistiques (APSA) variées pour former des citoyens cultivés, lucides, autonomes, physiquement et socialement éduqués dans le souci du vivre ensemble (MEN, 2015). Comme introduit dans notre première partie, les enseignants utilisent diverses stratégies pour arriver à leur fin de transmission et d'émancipation des élèves. Parmi elles, la méthode pédagogique Jigsaw peut permettre le développement de l'élève en ce sens en l'engageant et le motivant pour agir et apprendre. En effet, cette méthode peut permettre de structurer la classe pour rendre les élèves plus actifs dans leurs processus d'acquisition-production de connaissances (Cohen, 1994) grâce à l'observation, la pratique, la co-évaluation et le tutorat entre pairs (Escalié et al., 2018). Anderson, Christenson, Sinclair et Lehr (2004) soulignent que "l'engagement se produit lorsque les élèves sentent qu'ils peuvent interagir avec le contenu et ont le sentiment que leur vie est en quelque sorte " touchée " ou reflétée dans le contenu " (p. 95). Cet engagement est défini par la qualité des efforts déployés par les élèves pour réussir et atteindre les résultats souhaités ( $\mathrm{Hu} \& \mathrm{Kuh}, 2002$ ). L'engagement des élèves et les efforts consentis sont directement reliés à leur motivation. Celle-ci correspond à un "processus psychologique, neuronal et biologique non observable, privé qui sert d'antécédent causal au comportement publiquement observable qu'est l'engagement " (Reeve, 2012, p. 151). En tant qu'enseignant, réfléchir à des dispositifs permettant de motiver les élèves est essentiel. L'utilisation d'une structure d'enseignement comme le Jigsaw peut s'avérer un moyen efficace pour atteindre les visées communes de la discipline vers les élèves.

Le Jigsaw n'a pas fait l'objet d'une revue de littérature dans le domaine de l'éducation ni même plus particulièrement en EPS. Ainsi pour légitimer la méthode Jigsaw dans les pratiques pédagogiques actuelles et futures, nous devons aller au-delà de la notion intuitive que le Jigsaw " fonctionne " (Holliday, 2000; Maftei \& Maftei, 2011). Une analyse des travaux existants permet de comprendre comment et sous quelles conditions le Jigsaw propose des conditions pédagogiques bénéfiques en milieu scolaire. En s'appuyant sur les apports des recherches antérieures, l'objectif de cet article est d'étayer en quoi la méthode du Jigsaw pourrait être une approche intéressante en EPS.

\section{Méthode}

\subsection{Critères de sélection des articles}

Dans cette revue de littérature, seuls les articles scientifiques sur le Jigsaw dans le contexte éducatif ont été retenus. La recherche d'articles a été effectuée via les bases de données ERIC, Sciencesdirect, TaylorandFrancis, Web of Sciences, Cairn et Googleschoolar sans aucune limitation sur l'année de publication. Les combinaisons suivantes de mots clefs ont été réalisées : "Jigsaw», "Jigsaw éducation ", " Jigsaw éducation physique », "Jigsaw education", "Jigsaw physical education». La recherche s'est limitée à des articles de recherche en anglais et en français. Suite à cette recherche, les articles ont été analysés pour déterminer leur pertinence selon nos critères d'inclusion. Nous nous sommes limités à l'inclusion d'articles scientifiques obtenus à partir des bases de données, ainsi qu'aux articles scientifiques et ouvrages obtenus à partir des bibliographies contenues 
dans les documents initialement découverts. Au total, 79 articles ont été sélectionnés à partir du titre et du résumé.

\subsection{Analyse}

L'analyse des 79 études sélectionnées a suivi un processus systématique d'analyse inductive et de comparaison constante, selon les protocoles recommandés par Denzin et Lincoln (1994) et Lincoln et Guba (1985). Chaque étude a été lue en premier pour confirmer son inclusion initiale dans la revue de littérature et a été relue de manière indépendante et codée " to make the task of analysis more straightforward by sifting relevant materials from a large body of recording and transcript » (Potter, 2009, p. 615). Après cela, 57 études ont été conservées et font l'objet de notre corpus de littérature. Ce processus a permis de rassembler les études dans des sous-ensembles : par discipline, par résultats (positifs, pas de différence, négatifs), année de publication, niveau académique des élèves, nombre de sujets, pays d'étude, types de méthode Jigsaw et méthode de recherche employée (Tableau II). L'analyse plus fine des résultats a permis de faire émerger deux thèmes principaux : (1) la réussite des élèves en classe, et (2) les attitudes des élèves à l'égard de la méthode. L'identification de ces thèmes a été réalisée par deux chercheurs de manière indépendante et les résultats d'analyse comparés jusqu'à l'obtention d'un consensus.

Tableau II : description des études retenues dans la revue de littérature

\begin{tabular}{|c|c|}
\hline Catégories & $\mathrm{N}$ \\
\hline Date de publication & \\
\hline$>2000$ & 2 \\
\hline $2000-2010$ & 10 \\
\hline $2010-2015$ & 15 \\
\hline$<2015$ & 30 \\
\hline Échantillons & \\
\hline$>300$ & 3 \\
\hline$>100$ & 16 \\
\hline$>50$ & 17 \\
\hline$<50$ & 11 \\
\hline Continent & \\
\hline Afrique & 3 \\
\hline Amérique & 4 \\
\hline
\end{tabular}




\begin{tabular}{|c|c|}
\hline Asie & 36 \\
\hline Europe & 12 \\
\hline Non défini & 2 \\
\hline Niveau académique & \\
\hline Primaire & 6 \\
\hline Secondaire & 19 \\
\hline Université & 29 \\
\hline Formation enseignante & 3 \\
\hline Types de méthode Jigsaw & \\
\hline Jigsaw I & 45 \\
\hline Jigsaw II & 10 \\
\hline Jigsaw IV & 1 \\
\hline Jigsaw II et IV & 1 \\
\hline Durée de l'étude & \\
\hline$>10$ semaines & 11 \\
\hline 10-5 semaines & 11 \\
\hline$<5$ semaines & 18 \\
\hline Non défini & 17 \\
\hline Méthode de recherche & \\
\hline Avec groupe contrôle & 41 \\
\hline Sans groupe contrôle & 16 \\
\hline
\end{tabular}

\section{Résultats}

14 Les résultats de cette revue de littérature sont présentés en trois parties. Tout d'abord, nous développerons les résultats concernant la réussite des élèves en classe. Ensuite, nous détaillerons les résultats qui s'intéressent aux attitudes des élèves à l'égard de la méthode Jigsaw. Enfin, vis-à-vis de notre sujet d'étude qu'est l'EPS, nous développerons plus particulièrement les résultats des cinq études réalisées dans cette discipline scolaire. 


\subsection{La réussite des élèves en classe}

Au total, 41 études (71.93 \%) concernent la réussite des élèves en classe (Tableau III). Parmi elles, 31 (75.61 \% des 41 études) montrent que le Jigsaw a des effets positifs sur la réussite des élèves (Artut \& Tarim, 2007; Doymus, 2007; Er, 2017; Ghaith, 2003; Karacop \& Diken, 2017; Kardas et al., 2013). Six études (14.63 \%) ne montrent pas de différences significatives et quatre études $(9.76 \%)$ présentent des résultats mitigés, c'est-à-dire positifs et négatifs. Parmi les études s'intéressant à la réussite des élèves en classe, certaines se sont focalisées en partie sur des variables telles que le niveau d'habileté ou le sexe (Artut \& Tarim, 2017; Hamadneh, 2017; Yoruk, 2016).

Tableau III : analyse des résultats des études sur Jigsaw (positifs, négatifs, pas de différence) et comparaison selon la discipline scolaire, le niveau académique et le type de méthode Jigsaw utilisé.

\begin{tabular}{|c|c|c|c|c|}
\hline \multirow{2}{*}{ Thème } & \multicolumn{3}{|l|}{ Résultats } & \multirow[b]{2}{*}{$\begin{array}{l}\text { Résultats positifs } \\
\text { et négatifs }\end{array}$} \\
\hline & $\begin{array}{l}\text { Résultats } \\
\text { positifs }\end{array}$ & $\begin{array}{ll}\text { Pas de } \\
\text { différence }\end{array}$ & $\begin{array}{l}\text { Résultats } \\
\text { négatifs }\end{array}$ & \\
\hline \multicolumn{5}{|l|}{ Discipline scolaire } \\
\hline Sciences sociales & 4 & 1 & 0 & 0 \\
\hline Sciences de la vie & 21 & 3 & 3 & 4 \\
\hline Langues & 10 & 1 & 2 & 1 \\
\hline Éducation Physique & 2 & 0 & 0 & 3 \\
\hline Géographie & 1 & 0 & 0 & 0 \\
\hline Plusieurs disciplines scolaires & 1 & 1 & 0 & 1 \\
\hline $\mathrm{Nd}$ & 1 & 0 & 0 & 0 \\
\hline \multicolumn{5}{|l|}{ Niveau scolaire } \\
\hline Primaire & 4 & 1 & 1 & 0 \\
\hline Secondaire & 13 & 1 & 2 & 4 \\
\hline Université & 20 & 3 & 2 & 3 \\
\hline Formation enseignante & 3 & 0 & 0 & 0 \\
\hline \multicolumn{5}{|l|}{ Type de méthode } \\
\hline Jigsaw I & 30 & 4 & 5 & 5 \\
\hline Jigsaw II & 8 & 0 & 0 & 1 \\
\hline Jigsaw IV & 1 & 1 & 0 & 1 \\
\hline
\end{tabular}




\begin{tabular}{|l|l|l|l|l|}
\hline Jigsaw II et IV & 1 & 0 & 0 & 0 \\
\hline Thèmes des études & & & & \\
\hline La réussite des élèves en classe & 31 & 6 & 0 & 4 \\
\hline $\begin{array}{c}\text { Les attitudes des élèves à } \\
\text { l'égard de la méthode }\end{array}$ & 11 & 1 & 0 & 4 \\
\hline
\end{tabular}

Dans la majorité des études analysées, les élèves ont eu une meilleure compréhension des activités menées en classe, leurs connaissances ont augmenté et leur rétention a été favorisée (Doymus, 2008; Escalié, Legrain \& Lafont, 2018; Karacop \& Diken, 2017; Van Dat, 2016). Les études de ce corpus ont été réalisées prioritairement en sciences et en langues (e.g., Aydin \& Biyikli, 2017; Karacop \& Diken, 2017; Maftei \& Maftei, 2011; Sahin, 2010; Yoruk, 2016) (Tableau III). Par exemple, l'étude de Karacop et Diken (2017) en sciences montre que les résultats au test de compétences scientifiques (Scientific Process Skills Test) sont significativement meilleurs pour le groupe expérimental que pour le groupe contrôle en post-test.

Certaines études ne montrent pas de différence significative entre le groupe en condition Jigsaw et le groupe en condition contrôle ( $14.63 \%$ des 41 études sur la réussite des élèves), comme celle de Maden (2011) réalisée en expression écrite (Success Test for Written Expression).

D'autres études montrent des résultats mitigés pour la réussite scolaire des élèves en classe (9.76\% des 41 études sur la réussite des élèves) (Tableau III), en effet, des résultats négatifs et positifs ont été observés. C'est le cas dans l'étude de Wilson, Pegram, Battise et Robinson (2017), en pharmaceutique qui selon les outils de recueil de données utilisés n'obtiennent pas de résultats allant dans le même sens. Les scores post-test sont significativement plus favorables à la méthode traditionnelle comparés à la méthode Jigsaw. Cependant, les étudiants ayant fait l'expérience du Jigsaw ont amélioré leurs compétences en résolution de problèmes dans les contenus étudiés ainsi que leurs compétences sociales notamment au niveau de l'écoute et de la communication.

19 À noter, que plusieurs études (7.31 \% des 41 études sur la réussite des élèves) se sont intéressées à savoir si le niveau d'habileté ou le sexe des élèves pouvaient avoir une influence sur l'expérience du Jigsaw en classe (e.g., Artut \& Tarim, 2017; Hamadneh, 2017; Yoruk, 2016). Les résultats sont identiques, quel que soit le sexe ou le niveau d'habileté. Par exemple, Artut et Tarim (2017) ont étudié plus spécifiquement l'influence du niveau d'habileté des élèves sur leur réussite. Ils ont comparé trois groupes d'élèves en fonction des scores obtenus sur un test de rendement réalisé avant la mise en place de la méthode Jigsaw : "élevé " (scores de 65 et plus), " moyen » (scores de 45 à 64) et " faible " (scores de 44 ou moins). Après l'expérience de la méthode Jigsaw, il y avait une amélioration des moyennes des sujets dans les trois groupes et aucune différence significative n'a été trouvée entre les groupes. De la même manière, aucune différence n'a été identifiée entre la réussite des filles et des garçons en sciences dans les études de Hamadneh (2017) et de Yoruk (2016). 


\subsection{Les attitudes des élèves à l'égard de la méthode} perception positive des étudiants de la présence d'un expert dans chaque groupe sur l'apprentissage, sur l'acquisition des connaissances et le travail harmonieux des membres du groupe, certains futurs enseignants du groupe Jigsaw ont déclaré s'ennuyer lors des expériences menées. D'autres ont émis l'opinion que les expériences étaient faciles à mener et qu'elles devraient être plus complexes. Enfin, ils ont indiqué que le nombre élevé d'expériences et le manque de temps affectaient leur apprentissage de manière négative.

\subsection{Les études réalisées en EPS}

Seulement cinq des 57 études du corpus ( $8.77 \%$, Tableau III) ont été réalisées en EPS (El-Basiony, 2015; Escalié, Legrain, \& Lafont, 2018; Legrain, Escalié, Lafont \& Chaliès, 2019; O'Leary et al., 2015; O'Leary \& Griggs, 2010). Ces études présentent des résultats nuancés par rapport à l'utilisation du Jigsaw sur la réussite des élèves et étudiants, ainsi que sur leurs attitudes à l'égard de la méthode employée. Les résultats d'El-Basiony (2015) montrent que les apprenants formés à l'aide de la stratégie d'apprentissage coopératif Jigsaw ont obtenu de meilleurs résultats que ceux qui ont reçu une méthode d'apprentissage conventionnelle. De plus, les étudiants en 
condition Jigsaw étaient plus disposés à travailler avec d'autres personnes sur les tâches qui leur étaient assignées et qu'ils fournissaient une aide et une assistance plus élaborées entre eux que leurs pairs en condition contrôle. L'étude d'Escalié et al. (2018) pointe également des résultats positifs. Les chercheurs se sont intéressés au sentiment d'efficacité personnelle d'étudiants sur la découverte d'une activité sportive en EPS : la savate boxe française. Les résultats montrent que les étudiants en condition Jigsaw " maîtrisent des connaissances règlementaires et des connaissances pour instruire à un niveau plus élevé » (p. 168) plus que les étudiants du groupe contrôle. Concernant le sentiment d'efficacité personnelle, les participants de l'étude d'Escalié et al. (2018) en condition Jigsaw ont manifestê un sentiment d'efficacité personnelle plus élevé que celui des étudiants en condition contrôle dans le cadre de la présentation verbale et de la démonstration d'une tâche. A contrario, Legrain et al. (2019) n'obtiennent pas de différences significatives selon leurs conditions concernant le sentiment d'efficacité personnelle. L'étude de Legrain et al. (2019) montre des différences significatives en termes de connaissances pédagogiques, pratiques et de compétences motrices en faveur des étudiants ayant fait l'expérience du Jigsaw comparé au groupe contrôle.

Les résultats des études d'o'Leary et Griggs (2010) et d'O'Leary, Wattison, Edward et Bryan (2015) sur l'apprentissage des étudiants avec la méthode Jigsaw en EPS à partir d'un modèle de recherche-action sont plus négatifs. Dans l'étude d'o'Leary, Wattison, Edward et Bryan (2015), trois futurs enseignants ont enseigné cette méthode à des élèves. Alors que dans celle d'O'Leary et Griggs (2010), 61 étudiants ont fait l'expérience du Jigsaw en gymnastique et 16 ont réellement participé à l'étude. Les résultats de ces deux études ont montré que les capacités pratiques limitées des élèves et des étudiants, leurs capacités pédagogiques minimales et leurs relations sociales problématiques avaient une incidence pas toujours positive sur l'apprentissage avec Jigsaw. Ainsi, l'engagement nuancé des élèves et des étudiants a eu un impact direct sur leurs apprentissages lors des leçons.

\section{Discussion}

26 Au regard des résultats présentés, la méthode Jigsaw présente un intérêt à être utilisée à l'école. Tout d'abord, nous développerons en quoi le Jigsaw est une méthode intéressante à développer en milieu scolaire. Puis, dans un deuxième temps, nous pointerons notre attention sur l'EPS. Enfin, nous nous intéresserons à la formation des enseignants pour l'utilisation de la méthode Jigsaw.

\subsection{Le Jigsaw, une méthode intéressante à développer en milieu scolaire}

D'après les études analysées, la méthode Jigsaw peut favoriser le bien-être des élèves en classe et dans leur vie quotidienne. Cette méthode stimule la motivation des élèves et augmente le plaisir de l'expérience d'apprentissage (Karacop \& Diken, 2017; Van Dat, 2016) en cherchant à faire émerger des apprenants actifs. L'efficacité de cette méthode peut être reliée au fait qu'elle permet de satisfaire les besoins psychologiques fondamentaux des élèves : l'autonomie, la compétence et l'appartenance sociale (Deci \& Ryan, 1985). La méthode Jigsaw permet aux élèves de devenir des apprenants actifs, responsables de leur propre apprentissage (Özdemir \& Arslan, 2016). Les élèves fixent 
leurs propres objectifs d'apprentissage, approfondissent leurs connaissances et font des efforts pour que leurs camarades acquièrent des connaissances théoriques qu'ils ont apprises (Maden, 2011). Ils sont guidés par les caractéristiques contextuelles de leur environnement dans un processus actif et constructif (Pintrich, 2000). Ainsi, en contexte Jigsaw, les élèves passent du temps à discuter activement et moins à écouter passivement (Colosi \& Zales, 1998). Ce dispositif permet de répondre au besoin d'autonomie des élèves et également au besoin d'appartenance sociale (Arslan, 2016). La méthode Jigsaw favorise la collaboration, l'interdépendance et le soutien entre pairs (Huang, 2000; Mari \& Gumel, 2015).

Par ailleurs, cette méthode permet également la réussite de tous les élèves. Les élèves les plus faibles sont favorisés puisqu'ils peuvent avancer au même rythme que le reste de la classe (Mengduo \& Xiaoling, 2010). De plus, selon le principe des expériences vicariantes (Bandura \& Walters, 1977), le fait d'observer un élève dans la réussite d'une tâche présumée difficile peut inciter ses partenaires à fournir les efforts nécessaires pour la réussir aussi (Lafont \& Moulin, 2006). Toutefois, l'une des craintes de la méthode Jigsaw est qu'elle puisse freiner les plus performants (Slavin, 1986). L'étude d'Artut et Tarim (2017) qui s'est intéressée à cela n'a montré aucune preuve que le Jigsaw affecterait différemment les élèves aux capacités différentes. Une relation interdépendante apparait entre l'autonomie, la coopération et la motivation (Buschs et al., 2012 ; Johnson \& Johnson, 1989). Plus cette relation est positive, plus elle permet le développement des compétences interpersonnelles et l'augmentation de la participation en classe. Les élèves connaissent leur groupe qui reste stable, ce qui génère une certaine sécurité affective. Les interactions sociales peuvent être à l'origine d'une élévation de la motivation et du niveau attentionnel (Lafont \& Moulin, 2006). Elles peuvent ainsi affecter directement la transformation de leurs comportements (Sahin, 2010). Cette méthode encourage un appui mutuel des uns avec les autres, plus que de l'enseignant lui-même. Dans cette méthode, la seule façon pour un élève d'apprendre d'autres compétences est d'écouter attentivement ses coéquipiers. Toutefois, ces interactions peuvent également avoir l'effet inverse et parasiter l'attention, l'engagement et la persévérance des élèves en situation (Nicholls, 1984). Ainsi, l'art et la manière d'interagir ne s'improvisent pas. Interagir avec autrui s'acquiert via des mises en situation où les élèves sont incités à comprendre ensemble ce qui leur est demandé, à essayer de trouver ensemble des stratégies de résolution, à mettre collectivement au clair leurs idées (Baudrit, 2005).

"L'organisation de la classe en groupe d'experts consiste à structurer le travail des élèves de façon à favoriser les interactions en tutorat réciproque, en vue de permettre des acquisitions disciplinaires, mais aussi méthodologiques et sociales" (Escalié, Legrain \& Lafont, 2017, p. 122). Le développement d'expertise dans des domaines particuliers chez les élèves permet de satisfaire leur besoin de compétence. Cette méthode les rend compétents pour intervenir dans leur apprentissage et celui d'autrui et leur donne également des responsabilités. Des compétences pour apprendre leur sont transmises et celles-ci participent à la réussite scolaire. Elles contribuent aux processus d'apprentissage tout au long de la vie des individus (Arslan, 2016).

30 Les apprentissages disciplinaires sont au cœur du processus. L'efficacité de cette méthode peut dépendre des types de contenus enseignés (Aydin \& Biyikli, 2017; Er, 2017; Escalié et al., 2015; Karacop \& Diken, 2017; Mengduo \& Xiaoling, 2010). Aucune étude n'a comparé l'efficacité de la méthode Jigsaw dans différentes disciplines 
scolaires, mais il semble que la matière enseignée puisse affecter la mise en place de cette méthode et son efficacité. La majorité des études a été réalisée en sciences. Cette discipline laisse la possibilité de mettre en place des expérimentations et de favoriser ainsi le travail collectif. Par exemple, dans l'étude d'Aydin et Biyikli (2017) en sciences, le contenu disciplinaire a été divisé en six expériences dans le groupe Jigsaw : (EXP.-1) Vitesse et Accélération, (EXP.-2) Chute libre, (EXP.-3) Inertie et masses gravitationnelles, (EXP.-4) Coefficient de frottement cinétique, (EXP.-5) Mouvement harmonique simple, (EXP.-6) Pendule simple (p. 1075). Au regard des résultats plus nuancés obtenus en EPS dans les cinq études à disposition, nous pouvons nous demander si le contenu enseigné et notamment l'APSA pratiquée n'a pas une influence directe sur l'efficacité de cette méthode. Nous détaillerons plus particulièrement ce point dans la partie suivante.

\subsection{Le Jigsaw, une méthode prometteuse en EPS sous certaines conditions}

31 Le Jigsaw semble une méthode intéressante à développer en EPS là où seulement cinq articles scientifiques existent à ce jour (El-Basiony, 2015; Escalié, Legrain, \& Lafont, 2018; Legrain, Escalié, Lafont \& Chaliès, 2019; O'Leary et al., 2015; O'Leary \& Griggs, 2010). Les études antérieures dans les autres branches comme en EPS ont permis de faire émerger quelques points négatifs ou conditions qui doivent être réunis pour que cette méthode soit efficace. Les relations sociales problématiques, les capacités pratiques limitées des élèves, leurs compétences pédagogiques réduites ou encore le temps d'apprentissage sont les points sensibles de cette méthode.

La méthode Jigsaw vise à améliorer les relations interpersonnelles et diminuer les conflits entre les élèves grâce aux interactions entre pairs et à l'interdépendance entre eux (Aronson, 1978). Néanmoins, les relations sociales peuvent être problématiques (Aronson, 1978; O'Leary et al., 2015). Les conflits ne sont pas à bannir selon Aronson (1978), car ils représentent une occasion permettant aux élèves de développer des habiletés à résoudre des problèmes. Il semble nécessaire que les enseignants soient prêts à consacrer du temps à la constitution des groupes. La gestion de l'hétérogénéité est un point clé de la méthode qui peut entrainer un dysfonctionnement rapide de celle-ci. Certains chercheurs comme Aronson (1978) et Metzler (2011) préconisent un regroupement hétérogène en contexte Jigsaw afin que les élèves les plus en difficulté puissent prendre davantage appui sur les élèves les plus en réussite. La formation de groupes hétérogènes doit tenir compte du développement social et cognitif des élèves et non seulement de leurs capacités pratiques ou psychomotrices (O'Leary et al., 2015).

Dans l'étude d'o'Leary et al. (2015), l'expérience limitée des élèves en gymnastique semble avoir eu un impact négatif sur leurs apprentissages. Certains élèves se sont retrouvés en difficulté lorsque les experts ont dû enseigner ce qu'ils venaient d'apprendre presque immédiatement après l'avoir appris. Les élèves ont plutôt l'habitude des méthodes d'apprentissage dirigées prioritairement par les enseignants. Former des élèves pour coopérer et collaborer avec leurs pairs (Metzler, 2011) et gérer leur apprentissage de manière autonome fait partie de l'organisation du dispositif Jigsaw. Ces compétences sociales se construisent et se développent, elles doivent donc faire l'objet d'une réflexion par l'enseignant à propos de la manière de les aborder en EPS (Epinoux, 2014). Toutefois, Baudrit (2005) précise que si l'étape d'apprendre à 
coopérer n'est pas faite par l'enseignant avant le travail coopératif (i.e., Jigsaw), elle se fera de toute façon lors du travail lui-même avec plus ou moins de temps et avec des conséquences directes sur le travail collectif, sur l'engagement et donc sur l'apprentissage. Ainsi si le travail en groupe peut s'avérer extrêmement fructueux, il peut aussi se révéler inefficace (Slavin, 2011). L'individu et le collectif étant interdépendants avec la méthode Jigsaw, l'apprentissage doit être guidé de manière progressive et optimale par la structure mise en place par l'enseignant qui reste le superviseur (Buchs, Lehraus \& Crahay, 2012; O'Leary et al., 2015). Investir les élèves dans ce cadre d'apprentissage coopératif est essentiel. Les apprenants doivent accepter leurs rôles et responsabilités (Dyson \& Rubin, 2003; O'Leary et al., 2015). En cas d'absence d'un des élèves, les pairs ne peuvent ni apprendre, ni partager ses connaissances. Cela crée alors un décalage avec les autres élèves et groupes de la classe. Si les tâches de coopération données aux membres du groupe ne sont pas suffisamment difficiles pour nécessiter un effort conjoint, les membres du groupe peuvent considérer leurs contributions individuelles comme inutiles (Buchs, Lehraus \& Crahay, 2012; Karacop \& Doymus, 2013; Voyles, Bailey, \& Durik, 2015). Ceux-ci peuvent alors être enclins à l'évasion sociale et au désengagement. Si tous les élèves n'ont pas conscience qu'ils affectent l'apprentissage de leurs camarades de manière directe, alors le dispositif perd de son efficacité. Ovens, Dyson et Smith (2012) recommandent aux enseignants de donner plus de temps aux élèves pour réfléchir à la façon d'enseigner à leurs pairs. Chaque membre du groupe doit avoir une capacité à maîtriser correctement le contenu afin de pouvoir expliquer correctement aux autres membres du groupe (Mattingly \& VanSickle, 1991). Chaque élève "peut s'adapter au niveau de compréhension de son partenaire parce qu'il partage avec lui une même culture, un même langage, une même expérience " (Marchive, 1997, p. 40). Une solution pourrait, par exemple, consister à permettre aux élèves de se rassembler en dehors de leurs groupes « d'origine " pour réfléchir et mettre en pratique la façon dont ils pourraient enseigner avant de retourner dans leur groupe d'appartenance. Aronson (1978) appelle cela des "groupes de contrepartie». Aussi, la taille du groupe doit être réduite (entre quatre et huit membres maximum), car plus la taille du groupe est petite, plus la responsabilité individuelle peut être grande (Johnson, Johnson, \& Stanne, 2000). Les résultats de l'étude d'o'Leary et Griggs (2010) montrent qu'un certain nombre d'élèves a été en mesure d'accepter la responsabilité partagée d'apprendre, exigée par le Jigsaw, grâce à une maturité sociale et affective nécessaire pour enseigner et être enseignée les uns aux autres (Metzler, 2005).

Utiliser le Jigsaw sur une longue période (pendant plusieurs séquences d'enseignement ou dans différentes branches d'enseignement) pourrait permettre aux élèves de se familiariser plus facilement avec cette nouvelle forme d'enseignement (Tarim \& Akdeniz, 2008) et de développer les compétences nécessaires pour engager un travail de groupe d'apprentissage coopératif efficace (Azmin, 2016). Les élèves doivent comprendre le fonctionnement en classe, ce qui peut prendre un peu de temps au début de la mise en place de cette méthode (Aronson, 1978). Ce dispositif demande une réorganisation des interventions en classe et il peut être difficile pour l'enseignant de dévoluer l'autorité. Dans cette démarche d'enseignement, l'enseignant n'est plus le seul détenteur du savoir à construire. Le dispositif contraint une posture particulière de l'enseignant, en retrait dans un premier temps afin d'observer sa classe fonctionner. Dans un deuxième temps, son intervention consiste à accompagner les élèves experts dans la construction de leur expertise motrice : «L'enseignant intervenant pour faire 
évoluer la situation en référence aux préoccupations défensives (i.e., protection, riposte)" (Escalié et al., 2018, p. 166). Enfin, dans un troisième temps, l'enseignant guide l'action de ses élèves pour favoriser les interactions coopératives, le partage d'expériences et de connaissances, tout en permettant à chacun de construire et d'enrichir ses compétences motrices. Aronson (1978) voit l'enseignant comme un gardien de l'apprentissage cultivant une atmosphère positive pour faire apprendre. Lorsque tout est en place et que les élèves ont compris le fonctionnement général de la classe, alors l'enseignant a plus de temps pour individualiser ses retours pédagogiques (Sahin, 2010). Mettre en place la méthode Jigsaw en EPS demande ainsi une réflexion et une préparation en amont, qui peuvent être largement récompensées par les bénéfices que cette méthode peut apporter aux élèves et à l'enseignant.

Par ailleurs, en EPS, l'expérience de la démarche Jigsaw diffère en fonction de l'activité physique, sportive et artistique (APSA) enseignée puisque toutes les APSA ne peuvent être traitées de la même manière. En effet, les contenus d'enseignement, les situations d'apprentissage et notamment les situations de référence diffèrent en fonction des APSA. D'après les résultats des études, il semblerait que certaines activités soient plus favorables à l'utilisation du Jigsaw en EPS. Par exemple, les deux études basées sur la gymnastique (O'Leary et al., 2015; O'Leary \& Griggs, 2010) mettent en avant des résultats mitigés quant à la mise en place du Jigsaw en classe. Dans ces deux études, les élèves étaient experts d'une habileté spécifique qu'ils devaient ensuite enseigner à leurs camarades (i.e., groupe d'appartenance). Ces derniers se sont retrouvés particulièrement en difficulté. Ceci peut s'expliquer en partie par l'activité pratiquée, la gymnastique dans ces deux études. Cette APSA est une activité morphocinétique qui est principalement vécue de manière individuelle. Ces études ont porté sur une démarche d'enseignement axée sur l'apprentissage de techniques gymniques; ce qui peut expliquer les difficultés rencontrées par les élèves dans cette séquence d'enseignement Jigsaw. Dans cette APSA, une possibilité serait de placer les élèves face à une situation de référence de prestation collective en interdépendance pour favoriser une dynamique de groupe et une réponse positive au but commun. Le respect des cinq critères de l'apprentissage coopératif (i.e., interdépendance positive, responsabilités individuelles et collectives, interactions en face à face, compétences sociales, dynamique de groupe avec un but commun) est indispensable pour voir la méthode Jigsaw fonctionner dans nombre d'APSA. Certaines APSA pourraient donc se prêter plus facilement à l'expérience du Jigsaw. Le relais-vitesse, par exemple permet de faire collaborer les élèves comme l'indique sa logique interne. En effet, les coureurs (deux minimum) doivent utiliser une vitesse optimale pour faire parcourir au témoin une distance donnée le plus vite possible en perdant un minimum de temps lors de la transmission. Les situations d'apprentissage et la situation de référence favorisent naturellement le développement de compétences sociales comme s'entraider ou apprendre à négocier pour s'organiser pour la course et prendre des risques pendant la transmission. De la même manière, «la logique interne de l'activité en sport collectif implique une coopération dans la tâche » (Darnis \& Lafont, 2008, p. 71). L'utilisation du Jigsaw dans ce type d'APSA doit être réfléchie puisque «dans le cas d'une situation de jeu collectif la dissymétrie ne peut pas être trop marquée, car l'interaction et la coopération dans la tâche motrice doivent être maintenues " (Darnis \& Lafont, 2008, p. 71). Il semble nécessaire de conserver la spécificité des APSA (i.e., logique interne, contenus d'enseignement, situations d'apprentissage, situation de référence) tout en 
faisant attention de ne pas favoriser une démarche trop techniciste afin de faciliter la coopération entre les élèves.

\subsection{Mieux former les enseignants à la méthode Jigsaw}

Dans le Jigsaw, le rôle de l'enseignant diffère d'un enseignement «frontal ». En effet, l'enseignant a un rôle de facilitateur, de guide. L'enseignant ne donnant plus les contenus de manière frontale, il peut utiliser son temps plus efficacement (Colosi \& Zales, 1998). Blatchford, Baines, Rubie-Davies, Basett et Chowne (2006) mettent en avant que le travail en petits groupes manque souvent de qualité puisque les enseignants manquent de formation et de préparation malgré un intérêt perceptible des étudiants en formation initiale (Escalié et al., 2018; Erdem, 2009). Ainsi, les enseignants ont besoin d'être formés aux méthodes d'apprentissage coopératif et notamment à la méthode Jigsaw. Peu d'études sont axées sur la formation de futurs enseignants à la méthode Jigsaw (Escalié et al., 2018; Legrain et al., 2019; Lloyd \& Frykholm, 2000; O'Leary et al., 2015). De plus, cette méthode n'est que très rarement, voire jamais abordée dans les formations actuelles des enseignants d'EPS, aussi bien au niveau de la formation initiale que continue. «Les étudiants ont rarement l'opportunité d'expérimenter au cours de leur formation initiale les dispositifs coopératifs d'apprentissage qu'ils sont censés mettre en œuvre ultérieurement en classe » (Escalié et al., 2018, p. 162). Il semble nécessaire de réfléchir plus en profondeur sur la meilleure façon de former les enseignants à la méthode Jigsaw, en utilisant notamment les résultats d'études antérieures. Comme indiqué par Artut et Tarim (2007), les compétences d'apprentissage coopératif doivent être modélisées et mises en pratique lors de la formation des enseignants pour préparer les futurs enseignants à l'utilisation de ces compétences en classe. Les études rapportent deux méthodes de formation pour les enseignants : la méthode Jigsaw testée par les futurs enseignants eux-mêmes lors des cours de formation, et l'utilisation de cette méthode par les étudiants en stage. Lorsque les futurs enseignants ont testé la méthode les uns avec les autres (Lloyd \& Frykholm, 2000), les APSA du groupe coopératif ont donné aux futurs enseignants l'occasion de réfléchir à la nature des contenus enseignés, de faire le lien entre leur propre apprentissage et celui de leurs futurs élèves (Artut \& Tarim, 2007). Avoir fait l'expérience en formation, ce type de méthode peut affecter la réussite scolaire (Artut \& Tarim, 2007) et favoriser leur sentiment d'efficacité personnelle des futurs enseignants (Escalié et al., 2018). De plus, expérimenter ces dispositifs permet selon les enseignants de mieux assister les élèves en difficulté dans leurs apprentissages et de développer plus facilement un climat motivationnel propice au travail (Abrami, Poulsen, Chambers, 2004 ; Escalié et al., 2018). Le but du développement professionnel est notamment « d'équiper les enseignants en nouvelles habiletés et expériences pour faire la classe" (Brody \& Davidson, 1998a, p. 4). La méthode Jigsaw permet le développement de connaissances pédagogiques (Legrain et al., 2019). Il est alors probable qu'ils reproduiront avec leurs propres élèves ce qu'ils ont appris et comment ils ont été enseignés. À ce jour, la littérature existante et le manque de travaux dans ce domaine ne permettent pas de mettre en avant une méthode de formation plus efficace qu'une autre. 


\section{Limites et perspectives}

37 Un certain nombre d'études a été réalisé en milieu scolaire sur le Jigsaw. Cette méthode semble prometteuse en EPS. Néanmoins, les travaux antérieurs présentent un certain nombre de limites qui amènent à proposer des pistes d'études futures.

Tout d'abord, les échantillons d'étude étaient relativement petits : 19,30\% des études avaient des échantillons de moins de 50 sujets et $29,82 \%$ des études avaient un échantillon compris entre 50 et 100 sujets. Il semble important de développer des études futures avec des échantillons plus larges et plus représentatifs afin de vérifier les effets du Jigsaw sur la réussite et l'engagement des élèves ainsi que sur leurs attitudes à l'égard de la méthode, tout en prenant en considération d'autres variables qui pourraient influencer ce résultat (e.g., sexe des élèves, niveau d'habileté).

Une deuxième limite est la répétition des séquences d'enseignement. Toutes les études de corpus n'ont travaillé que sur une séquence d'enseignement. La répétition des séquences d'enseignement est recommandée pour favoriser une routine automatique et offrir des avantages aux élèves en termes d'engagement et d'apprentissage. Pour évaluer les avantages à long terme en classe, il semble pertinent de proposer de futures recherches sur l'utilisation de la méthode au cours d'une année scolaire sur différentes séquences d'enseignement.

Ensuite, en EPS, seulement cinq études ont été réalisées et peu d'APSA ont donc été testées (e.g., gymnastique, savate boxe française). Face à ces limites, il semble intéressant de mettre en place une étude quantitative et longitudinale sur un grand nombre d'élèves et sur différentes séquences d'enseignement avec des APSA variées utilisant la démarche Jigsaw en EPS. Ceci permettra de mesurer les effets de la méthode Jigsaw en EPS sur l'engagement des élèves en prenant en considération la spécificité des APSA enseignées.

41 Le manque de formation (e.g. en formation initiale et en formation continue) des enseignants a été mis en avant dans cette revue de littérature, et apparait comme une limite au développement et à l'utilisation de la méthode Jigsaw dans le milieu éducatif. Ainsi, le développement professionnel et l'évolution des conceptions d'enseignement pourraient être étudiés en lien avec la formation et la mise en place de cette approche par les enseignants.

\section{Conclusion}

Dans un contexte où l'intérêt pour la vie en commun et la coopération augmente à l'école (Casey \& Goodyear, 2015), les enseignants et les personnels de l'éducation ne cessent de se demander : comment pouvons-nous apprendre mieux ? (Aydin \& Biyikli, 2017). L'objectif de cet article était d'étayer en quoi le Jigsaw peut être une méthode intéressante en EPS au regard des résultats des recherches antérieures. Le Jigsaw est une méthode d'apprentissage coopératif qui a prouvé son efficacité en milieu scolaire. Cet article permet de mettre en évidence les conditions dans lesquelles la méthode est efficace. Il permet également de souligner le manque de travaux sur le Jigsaw en EPS (cinq études). Certains chercheurs (Haerens, Kirk, Cardon, \& De Bourdeaudhuij, 2011; Kirk, 2010; 2013) soutiennent que l'EPS devrait promouvoir une approche globale et cohérente des apprentissages au niveau physique, cognitif, social et affectif. La 
méthode Jigsaw permet de satisfaire les besoins fondamentaux chez les élèves (Deci \& Ryan, 1985) et est un moyen d'offrir une expérience d'apprentissage holistique aux apprenants en EPS (Barrett, 2005; Dyson, 2002; Dyson \& Grineski, 2001). Cette méthode mérite donc qu'on s'y intéresse davantage en EPS.

\section{BIBLIOGRAPHIE}

Abrami, P. C., Poulsen, C., \& Chambers, B. (2004). Teacher motivation to implement an educational innovation: factors differentiating users and non-users of cooperative learning. Educational Psychology, 24, 201-216.

Anderson, A. R., Christenson, S. L., Sinclair, M. F., \& Lehr, C. A. (2004). Check \& Connect: The importance of relationships for promoting engagement with school. Journal of School Psychology, 42(2), 95-113.

Aronson, E. (1978). The jigsaw classroom. Beverly Hills, CA: Sage.

Arslan, A. (2016). Effect of Jigsaw I technique on teaching Turkish grammar. Educational Research and Reviews, 11(8), 635.

Artut, P. D., \& Tarim, K. (2007). The effectiveness of jigsaw II on prospective elementary school teachers. Asia-Pacific Journal of Teacher Education, 35(2), 129-141.

Aydin, A., \& Biyikli, F. (2017). The Effect of Jigsaw Technique on the Students' Laboratory Material Recognition and Usage Skills in General Physics Laboratory-I Course. Universal Journal of Educational Research, 5(7), 1073-1082.

Azmin, N. H. (2016). Effect of the Jigsaw-Based Cooperative Learning Method on Student Performance in the General Certificate of Education Advanced-Level Psychology: An Exploratory Brunei Case Study. International Education Studies, 9(1), 91-106.

Bandura, A., \& Walters, R. H. (1977). Social learning theory (Vol. 1). Englewood Cliffs, NJ: Prenticehall.

Barrett, T. (2005). Effects of cooperative learning on performance of sixth-grade physical education students. Journal of Teaching in Physical Education, 24(1), 88-102.

Baudrit, A. (2005). L'apprentissage coopératif: origines et évolutions d'une méthode pédagogique. Bruxelles: De Boeck.

Blatchford, P., Baines, E., Rubie-Davies, C., Bassett, P., \& Chowne, A. (2006).The effect of a new approach to group work on pupil-pupil and teacher-pupil interactions. Journal of Educational Psychology 98(4), 750-765.

Brody, C. M., \& Davidson, N. (1998). Professional development for cooperative learning: issues and approaches. Albany, NY: State University of New York Press.

Buchs, C., Lehraus, K. \& Crahay, M. (2012). 8 - Coopération \& apprentissage. In M. Crahay (Eds.), L'école peut-elle être juste et efficace : De l'égalité des chances à l'égalité des acquis (pp. 421-454). Louvain-la-Neuve, Belgique: De Boeck Supérieur. 
Byra, M. (2006). Teaching styles and inclusive pedagogies. In D. Kirk, D. MacDonald, \& M. O'Sullivan (Eds.), The handbook of physical education (pp. 449-466). London: Sage Publications.

Casey, A. (2004). Piece-by-piece cooperation: pedagogical change and jigsaw learning. British Journal of Teaching Physical Education, 35(4), 11-12.

Casey, A., \& Goodyear, V. (2015). Can cooperative learning achieve the four learning outcomes of physical education? A review of literature. Quest, 67(1), 56-72.

Cohen E. G. (1994). Restructuring the classroom: Conditions for productive small groups. Review of Educational Research, 64, 1-35.

Colosi, J. C., \& Zales, C. R. (1998). Jigsaw cooperative learning improves biology lab courses. Bioscience, 48(2), 118-124.

Darnis, F., \& Lafont, L. (2008). Effets de la dissymétrie de compétence pour un apprentissage coopératif en dyades en Éducation Physique et Sportive. Les Cahiers Internationaux de Psychologie Sociale 79, 69-83.

Deci, E. L., \& Ryan, R. M. (1985). The general causality orientations scale: Self-determination in personality. Journal of Research in Personality, 19(2), 109-134.

Denzin, N. K., \& Lincoln, Y. S. (1994). Handbook of qualitative research (Vol. 2). Thousand Oaks, CA: Sage.

Deutsch, M. (1949). A theory of cooperation and competition. Human Relations, 2, 129-152

Doymus, K. (2007). Effects of a cooperative learning strategy on teaching and learning phases of matter and one-component phase diagrams. Journal Chemistry Education, 84(11), 1857.

Doymus, K. (2008). Teaching chemical equilibrium with the jigsaw technique. Research in Science Education, 38(2), 249-260.

Dyson, B. (2002). The implementation of cooperative learning in an elementary physical education program. Journal of Teaching in Physical Education, 22(1), 69-85.

Dyson, B., \& Casey, A. (2013). Cooperative Learning in Physical Education. A research-based approach. Oxon: Routledge.

Dyson, B., \& Grineski, S. (2001). Using cooperative learning structures in physical education. Journal of Physical Education, Recreation \& Dance, 72(2), 28-31.

Dyson, B., \& Rubin, A. (2003). Implementing cooperative learning in elementary physical education. Journal of Physical Education, Recreation \& Dance, 74(1), 48-55.

Er, H. (2017). The Impact of Teaching the Subjects under" Science in Time" Unit in the Social Studies Class in the 7th Grade Using Jigsaw Technique on the Academic Success of the Students. Universal Journal of Educational Research, 5(5), 838-847.

Erdem A. (2009). Preservice teachers' attitudes towards cooperative learning in mathematics course. Procedia Social and Behavioral Sciences, 1, 1668-1672.

Epinoux, N. (2014). Les compétences sociales et l'apprentissage coopératif au collège : enjeux et perspectives. Apprendre à coopérer pour réaliser un projet collectif en EPS et en Sciences Physiques (Thèse de doctorat en STAPS soutenue le 12 décembre 2014). Université de Bordeaux.

El-Basiony, A. (2015). Effect of Jigsaw instructional method on pre-Service teacher teaching proficiency skills and perceptions toward working in small-groups. Journal of Applied Sports Science, 5, 108-115. 
Escalié, G., Legrain, P., \& Lafont, L. (2017). Travailler ensemble au cycle 3 : renforcer la collaboration des enseignants au service des acquisitions des élèves. Colloque Pratiques sociales et apprentissages. Saint-Denis, 8-9 Juin 2017.

Escalié, G., Legrain, P., \& Lafont, L. (2018). L'apprentissage coopératif en «groupe d'experts» et la professionnalisation des futurs enseignants: un exemple en éducation physique et sportive. Carrefours de l'éducation, 46, 161-176.

Evin, A. (2013). Coopération entre élèves et histoires collectives d'apprentissage en Éducation Physique et Sportive Contribution à la compréhension des interactions entre élèves et au développement de dispositifs d'apprentissage coopératif. Thèse de doctorat en STAPS soutenue le 6 décembre 2013, Université de Nantes.

Ghaith, G. M. (2003). Relationship between reading attitudes, achievement, and learners perceptions of their Jigsaw II cooperative learning experience. Reading Psychology, 24(2), 105-120.

Haerens, L., Kirk, D., Cardon, G., \& De Bourdeaudhuij, I. (2011). Toward the development of a pedagogical model for health-based physical education. Quest, 63(3), 321-338.

Hamadneh, Q. M. S. (2017). The Effect of Using Jigsaw Strategy in Teaching Science on the Acquisition of Scientific Concepts among the Fourth Graders of Bani Kinana Directorate of Education. Journal of Education and Practice, 8(5), 127-134.

Hamel, J. (1818). L'enseignement mutuel ou histoire de l'introduction et de la propagation de cette méthode. Paris : Colas.

Hameline, D. (1994). Qu'est-ce qu'une Idée Pédagogique? Pour une Philosophie de l'Éducation. Actes du colloque Philosophie de l'éducation et formation des maîtres, Dijon, France.

Hänze, M., \& Berger, R. (2007). Cooperative learning, motivational effects, and student characteristics: An experimental study comparing cooperative learning and direct instruction in 12th grade physics classes. Learning and Instruction, 17(1), 29-41.

Holliday, D. C. (2000). The Development of Jigsaw IV in a Secondary Social Studies Classroom. Paper presented at the Midwest Educational Research Association (MWERA) Annual Conference, Chicago, IL.

Hu, S., \& Kuh, G. D. (2002). Being (dis) engaged in educationally purposeful activities: The influences of student and institutional characteristics. Research in Higher Education, 43(5), 555-575.

Huang, C.-Y. (2000). The effects of cooperative learning and model demonstration strategies on motor skill performance during video instruction. Proceeding National Sciences Council, 2, 255-268.

Jaakkola, E., Helkkula, A., \& Aarikka-Stenroos, L. (2015). Service experience co-creation: conceptualization, implications, and future research directions. Journal of Service Management, 26(2), 182-205.

Johnson, D., \& Johnson, R. (1989). Cooperation and competition: Theory and research. Edina: MN : Interaction Book Company.

Johnson, D., Johnson, R., Holubec, E., \& Roy, P. (1993). Circles of learning. Edina: MN: Interaction Book Company.

Johnson, D. W., Johnson, R. T., \& Stanne, M. B. (2000). Cooperative learning methods: A meta-analysis. Minneapolis: University of Minnesota.

Kagan, S., \& Kagan, M. (2009). Kagan cooperative learning. San Clemente, CA: Kagan. 
Karacop, A., \& Diken, E. H. (2017). The Effects of Jigsaw Technique Based on Cooperative Learning on Prospective Science Teachers' Science Process Skill. Journal of Education and Practice, 8(6), 86-97.

Karacop, A., \& Doymus, K. (2013). Effects of jigsaw cooperative learning and animation techniques on students' understanding of chemical bonding and their conceptions of the particulate nature of matter. Journal of Science Education and Technology, 22(2), 186-203.

Kardas, P., Lewek, P., \& Matyjaszczyk, M. (2013). Determinants of patient adherence: a review of systematic reviews. Frontiers in Pharmacology, 4, 91.

Kirk, D. (2010). Physical education futures. London, England: Routledge.

Kirk, D. (2013). Educational value and models-based practice in physical education. Educational Philosophy and Theory, 45(9), 973-986.

Lafont, L., \& Moulin. (2006). Adolescence, estime de soi et interactions sociales. Revue Hyper-EPS, $234,2-4$.

Lafont L., \& Winnykamen F. (1999). Cooperation and Competition in children and adolescents: current issues and perspectives in physical education. In Y. Van den Auweele, F. Bakker, S. Biddle, M. Durand, R. Seiler (coord.), Textbook on psychology for physical educators (pp. 379-404). Champaign IL: Human Kinetics.

Legrain, P., Escalié, G., Lafont, L., \& Chaliès, S. (2019) Cooperative learning: a relevant instructional model for physical education preservice teacher training? Physical Education and Sport Pedagogy, 24(1), 73-86.

Legrain P., \& Heuzé J. - P. (2006). Favoriser les apprentissages en interactions de tutelle réciproque entre pairs en EPS : une compétence de l'enseignant à former les élèves. Colloque international de l'ARIS : Co-construire des Savoirs. Les métiers de l'intervention dans les APS. Besançon, 9-12 mai.

Lincoln, Y. S., \& Guba, E. G. (1985). Naturalistic inquiry. London, England: Sage.

Lloyd, G. M., \& Frykholm, J. A. (2000). How innovative middle school mathematics can change prospective elementary teacher's conception. Education, 120(3), 575-575.

Maden, S. (2011). Effect of Jigsaw I Technique on Achievement in Written Expression Skill. Educational Sciences: Theory and Practice, 11(2), 911-917.

Maftei, G., \& Maftei, M. (2011). The strengthen knowledge of atomic physics using the "mosaic" method (The Jigsaw method). Procedia-Social and Behavioral Sciences, 15, 1605-1610.

Marchive, A. (1997). L'interaction de tutelle entre pairs: approche psychologique et usage didactique. Psychologie et Éducation, 30, 29-42.

Mari, J., \& Gumel, S. A. (2015). Effects of jigsaw model of cooperative learning on self-efficacy and achievement in chemistry among concrete and formal reasoners in colleges of education in Nigeria. International Journal of Information and Education Technology, 5(3), 196.

Mattingly, R. M., \& VanSickle, R. L. (1991). Cooperative Learning and Achievement in Social Studies: Jigsaw II. Social Education, 55(6), 392-395.

Meirieu, P. (2013). Pédagogie : des lieux communs aux concepts clés. Paris, ESF éditeur.

MEN (2015). Programme d'éducation physique et sportive [en ligne], paru au BO $n^{\circ} d u 26$ novembre 2015, 294-300. http://cache.media.education.gouv.fr/file/MEN_SPE_11/35/1/ BO_SPE_11_26-11-2015_504351.pdf 
Mengduo, Q., \& Xiaoling, J. (2010). Jigsaw Strategy as a Cooperative Learning Technique: Focusing on the Language Learners. Chinese Journal of Applied Linguistics (Foreign Language Teaching \& Research Press, 33(4), 113-125.

Metzler, M. (2011). Instructional models for physical education (3rd ed.). Scottsdale, AZ: Holcomb, Hathway.

Nicholls, J. G. (1984). Achievement motivation: Conceptions of ability, subjective experience, task choice, and performance. Psychological Review, 91(3), 328-346.

O'Leary, N., \& Griggs, G. (2010). Researching the pieces of a puzzle: the use of a jigsaw learning approach in the delivery of undergraduate gymnastics. Journal of Further and Higher Education, 34(1), 73-81.

O'Leary, N., Wattison, N., Edwards, T., \& Bryan, K. (2015). Closing the theory-practice gap: physical education students' use of jigsaw learning in a secondary school. European Physical Education Review, 21(2), 176-194.

Ovens, A., Dyson, B., \& Smith, W. (2012). Implementing the cooperative learning model in physical education: the experience of the New Zealand teachers. In B. Dyson, A. Casey (Eds.), Cooperative learning in physical education (pp. 15-26). New York, NY: Routledge.

Özdemir, E., \& Arslan, A. (2016). The Effect of Self-regulated Jigsaw IV on University Students' Academic Achievements and Attitudes towards English Course. Journal of Education and Training Studies, 4(5), 173-182.

Piaget, J. (1969). Les méthodes nouvelles, leurs bases psychologiques. Psychologie et Pédagogie, 26, 4-16.

Piaget, J. (1971). Méthodologie des relations interdisciplinaires. Archives de Philosophie, 539-549.

Pintrich, P. R. (2000). Multiple goals, multiple pathways: The role of goal orientation in learning and achievement. Journal of Educational Psychology, 92(3), 544.

Potter, J. (2009). Discourse analysis. In M. Hardy, A. Bryman (Eds.), The handbook of data analysis, (pp. 607-624). London, England: Sage.

Reeve, J. (2012). A self-determination theory perspective on student engagement. In S.

Christenson, A.L. Reschly, C. Wylie (Eds.), Handbook of research on student engagement (pp. 149-172). New York, NY: Springer.

Sahin, A. (2010). Effects of jigsaw II technique on academic achievement and attitudes to written expression course. Educational Research and Reviews, 5(12), 777.

Slavin, R. E. (1986). Best-evidence synthesis: An alternative to meta-analytic and traditional reviews. Educational Researcher, 15(9), 5-11.

Slavin, R. E. (1996). Research on Cooperative Learning and Achievement: What We Know, What We Need to Know. Contemporary Educational Psychology, 21(1), 43-69.

Slavin, R.E. (2011). Instruction based on cooperative learning. In R. Mayer (Ed.), Handbook of research on learning and instruction (pp. 358-374). London: Taylor \& Francis.

Slavin, R. E. (2014). Cooperative learning and academic achievement: Why does groupwork work? Anales de Psicología/Annals of Psychology, 30(3), 785-791.

Stahl, R. J. (1994). The essential elements of cooperative learning in the classroom. Bloomington. 
Tarim, K., \& Akdeniz, F. (2008). The effects of cooperative learning on Turkish elementary students' mathematics achievement and attitude towards mathematics using TAI and STAD methods. Educational studies in Mathematics, 67(1), 77-91.

Topping K. J., \& Ehly S. (1998). Peer-Assisted Learning. New Jersey: Lawrence Elbaum Associates.

Van Dat, T. (2016). The Effects of Jigsaw Learning on Students' Knowledge Retention in Vietnamese Higher Education. International Journal of Higher Education, 5(2), 236.

Vygotsky, L. S. (1978). Mind in society: The development of higher psychological processes. Cambridge, MA: Harvard University Press.

Voyles, E. C., Bailey, S. F., \& Durik, A. M. (2015). New pieces of the jigsaw classroom: increasing accountability to reduce social loafing in student group projects. The New School Psychology Bulletin, 13(1), 11-20.

Williams, D. (2004). Improving race relations in higher education: The jigsaw classroom as a missing piece to the puzzle. Urban Education, 39(3), 316-344.

Yoruk, A. (2016). Effect of Jigsaw Method on Students' Chemistry Laboratory Achievement. International Journal of Educational Sciences, 15, 377-381.

Zach, S., \& Cohen, R. (2012). Using the Cooperative Learning model in physical education teacher education. In B. Dyson, A. Casey (Eds.), Cooperative Learning in Physical Education: A Research Based Approach (pp. 104-116). Routledge.

\section{RÉSUMÉS}

Cet article porte sur l'utilisation de la méthode d'apprentissage coopératif, le Jigsaw en éducation physique et sportive. En s'appuyant sur les apports des recherches antérieures, l'objectif de cet article est d'étayer en quoi la méthode Jigsaw peut être une approche intéressante en éducation physique et sportive. À ce jour, seulement cinq études ont porté sur son utilisation en éducation physique et sportive. Pourtant au regard des 57 études existantes analysées, cette approche semble prometteuse à mettre en place en éducation physique et sportive. Les travaux antérieurs montrent des résultats positifs sur l'engagement et l'apprentissage des élèves, ainsi que les perceptions des élèves et enseignants qui ont vécu des expériences Jigsaw dans le milieu éducatif. Des progrès sont à réaliser pour mettre en place ce type de méthode en éducation physique et sportive et dans les autres disciplines notamment en améliorant la formation des enseignants dans ce domaine.

The present paper focuses on the use of Jigsaw, a cooperative learning method, in Physical Education. Based on the findings of previous researches, the purpose of this paper is to discuss if and how the Jigsaw method could be valuable in Physical Education. To date, only five studies have focused on its use in Physical Education. However, considering the numerous existing studies analyzed in education $(\mathrm{N}=57)$, this approach seems appropriate to be implemented in Physical Education. The literature review in education has shown positive results of Jigsaw on students' engagement and learning, as well as on students' and teachers' perceptions. Progress needs to be made to implement this type of method in physical education and in other school subjects, notably by increasing teacher training in this area. 
INDEX

Mots-clés : Jigsaw, apprentissage coopératif, groupe de travail, éducation physique, engagement

Keywords : Jigsaw, cooperative learning, work group, physical education, engagement

\section{AUTEURS}

\section{OCÉANE DROUET}

Haute École Pédagogique du Canton de Vaud, Lausanne, Suisse

\section{GRÉGOIRE MILLET}

Institut des Sciences du Sport de l'Université de Lausanne (ISSUL), Lausanne, Suisse

\section{VANESSA LENTILLON-KAESTNER}

Haute École Pédagogique du Canton de Vaud, Lausanne, Suisse 